\title{
Retrospective Comparison of Landmark Based and Ultrasound Guided Suprascapular Nerve Steroid Injections in a Patient
}

\author{
Hariharan Shankara, d, Jaymin Shah ${ }^{\mathrm{b}}$, Dan Eastwood ${ }^{\mathrm{c}}$
}

\begin{abstract}
Background: Suprascapular nerve steroid injections are performed for the relief of chronic shoulder pain. This retrospective comparison between landmark based and ultrasound guidance was undertaken to see the advantages of using ultrasound guidance for the performance of these injections.
\end{abstract}

Methods: After institutional review board approval a chart review of all the suprascapular nerve steroid injections performed between 2005 and 2009 was done. Statistical analysis was performed using T-test and Wilcoxon Rank-Sum test and Mann-Whitney test for the confidence interval.

Results: There were a total of 12 suprascapular nerve steroid injections performed. The mean decrease in VAS from pre-procedure for the landmark based was $-2.67 \pm 0.577$ and for ultrasound guidance was $-4.50 \pm 1.173$. There was a statistically significant difference of changes in VAS between the landmark based and ultrasound guidance $(\mathrm{P}=0.0409)$. The median difference in the change in VAS was 2 points, and the $95 \%$ median confidence interval was 0.0 and 3.0. In addition, there was a $50 \%$ reduction in the volume of injectate and dose of methylprednisolone using the ultrasound guidance technique.

Conclusions: This study shows that there is a possibility for using lesser injectate volume and steroid for suprascapular nerve blocks

Manuscript accepted for publication April 29, 2011

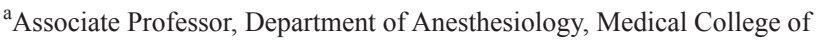
Wisconsin \& Clement Zablocki VA Medical Center, Milwaukee, Wisconsin, USA

${ }^{\mathrm{b}}$ Senior Resident, Department of Anesthesiology, Medical College of Wisconsin, Milwaukee, Wisconsin, USA

${ }^{\mathrm{c}}$ Biostatistician, Dept. of Biostatistics, Medical College of Wisconsin, Milwaukee, Wisconsin, USA

${ }^{\mathrm{d}}$ Corresponding author: Hariharan Shankar MBBS, Clement Zablocki VA Medical Center, 5000, West National Avenue, Milwaukee, Wisconsin 53295, USA, Email: hshankar@mcw.edu
}

doi:10.4021/jnr19e along with a marginal increase in pain relief with ultrasound guidance. Larger prospective studies are needed to further validate the utility of ultrasound guidance in chronic shoulder pain.

Keywords: Ultrasound; Suprascapular nerve; Shoulder pain

\section{Introduction}

Shoulder pain is a common complaint associated with significant pain and disability [1]. Conditions leading to shoulder pain include degenerative diseases of the gleno-humeral and acromio-clavicular joints, entrapment injuries in sports, trauma and shoulder dislocations, rheumatoid arthritis and other arthropathies. The safety and efficacy of suprascapular nerve (SSN) block with local anesthetics alone and with steroids in treating chronic shoulder pain and improving disability and range of motion is well documented [2-4]. SSN blocks have been used both in regional anesthesia and in chronic shoulder pain of varied etiology $[2,5]$. Besides the use of steroids for providing longer pain relief, the use of phenol and pulsed radiofrequency lesioning has also been described $[6,7]$. Most studies in the literature utilize landmark based injections. Electromyography, ultrasound and nerve stimulator guided approaches have also been described for SSN block [8-11]. Ultrasound imaging has advantages of portability, lack of radiation and ability to visualize pleura and vascular structures. At our institution we have been using ultrasound guidance in the pain clinic for blocking the SSN for the last few years. We undertook this pilot study of retrospective comparison of anatomic landmark based (AL) vs. ultrasound guided (USG) suprascapular nerve injection for chronic shoulder pain to look for any advantages to using ultrasound guidance for blocking the SSN.

\section{Methods}

Following institutional review board approval, we performed a chart review of the SSN blocks performed at our institution. The technique used and the initial and post pro- 


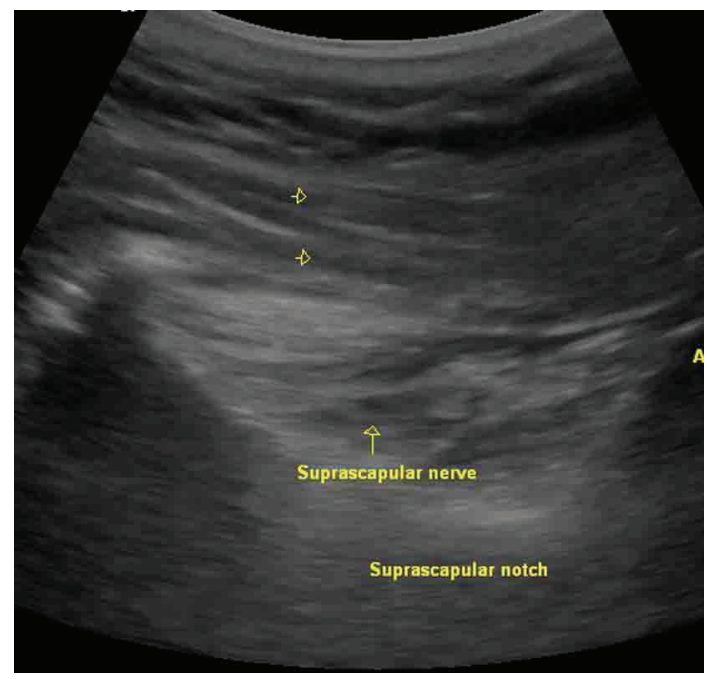

Figure 1. Sonographic picture showing the suprascapular nerve in the notch with arrowheads pointing to the 25 G spinal needle.

cedure VAS pain scores were recorded besides the duration of pain relief when available. The volume and contents of the injectate were also recorded from the chart.

\section{Techniques}

AL technique involved insertion of a sterile 22 G 3.5 inch spinal needle at a point approximately $1 / 3$ rd of the way along the scapular spine as originally described by Moore [12]. The point of entry was determined just lateral to the point of bisection of a line along the scapular spine and another from the inferior tip of the scapula to meet the scapular spine line. The needle was advanced to the bone and moved laterally into the suprascapular notch to elicit paraesthesia. The local anesthetic and methylprednisolone mixture were then injected after negative aspiration.

USG technique involved using a 10-5 MHz multi-frequency broadband, $38 \mathrm{~mm}$ linear array transducer in a sterile sheath to scan the scapula from the scapular spine upwards. Once the suprascapular artery was located the probe was adjusted to visualize the notch and the hyperechoic suprascapular nerve in proximity to the vessels. The technique was subsequently modified to scan from the acromion, moving medially and adjusting the tilt to visualize the notch base. Using a $25 \mathrm{G}$ hypodermic 3.5 inch needle in an out of plane approach the needle was advanced to the target. Methylprednisolone and local anesthetic were injected under real time imaging (Fig. 1).

No sedation was used for either technique.

\section{Statistical methods}

T-test and Wilcoxon Rank-Sum tests were performed using SAS version 9.2 (The SAS Institute, Cary, NC). Mann-Whit-

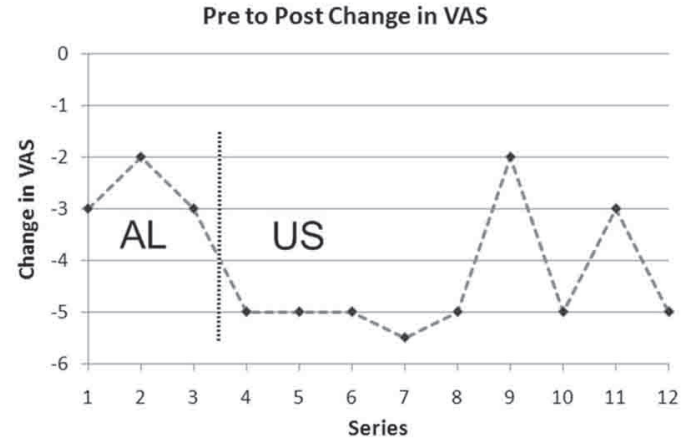

Figure 2. Chart showing the pre and post procedure VAS scores. AL: anatomic landmark based injections, US: ultrasound guided injections.

ney confidence interval was performed with the Minitab version 12.0 (Minitab, Inc., State College, PA).

\section{Results}

There were a total of 12 SSN steroid blocks performed. Three blocks were performed with anatomic landmarks and 9 using ultrasound guidance. All the blocks were performed on one patient who developed chronic right shoulder pain following a major shoulder reconstruction and fusion following a crush injury during combat. As the ability to identify the suprascapular notch with ultrasound in this patient was not convincing, fluoroscopic confirmation was used once along with ultrasound guidance. This revealed that his notch was relatively shallow, a normal anatomical variant.

The mean decrease in VAS from pre-procedure for the AL was $-2.67 \pm 0.577$ and for the USG was $-4.50 \pm 1.173$ (Fig. 2). There was a statistically significant difference of changes in VAS between the AL and USG $(\mathrm{P}=0.0409)$ by the Wilcoxon Rank Sum test. The median difference in the change in VAS was 2 points, and the Mann-Whitney 95\% median confidence interval was $(0.0,3.0)$. In addition, there was a $50 \%$ reduction in the volume of injectate and dose of methylprednisolone using the USG technique. Due to personal logistic reasons, the patient would return for repeat blocks only every 3 months and reported approximately 2 months duration of pain relief. There were no complications reported with either technique. Following this the patient received an ultrasound guided pulsed radiofrequency ablation of the suprascapular nerve with resolution of his shoulder pain for over 2 years.

\section{Discussion}

Although it is hard to draw conclusions from a small sample size, this retrospective comparison of AL and USG for SSN 
blockade suggests a $50 \%$ decrease in the volume of injectate and steroid along with a decrease in VAS scores using ultrasound guidance.

The shoulder joint is supplied primarily by axillary nerve and SSN with smaller branches from the subscapular and lateral pectoral nerves. SSN originates from the upper trunk with contributions from C5-6 and some variable contribution from $\mathrm{C} 4$. It travels anterior to the trapezius and parallel to the omohyoid, crosses the posterior triangle to enter the suprascapular notch. The superior articular branch comes off about $4.5 \mathrm{~cm}$ proximal to the transverse scapular ligament (TSL) but continues along with the main nerve beneath the ligament [13]. The TSL spans the suprascapular notch. This location is thought to be a common site for injuries to SSN besides the spinoglenoid notch. The SSN then travels towards the spine where it sends a branch to the supraspinatus muscle and winds around the spinoglenoid notch to supply the infraspinatus. In its course along the scapular spine, the inferior articular branch separates from the main nerve and courses obliquely to supply the posterior shoulder joint [13]. SSN supplies $70 \%$ of the sensory fibers to the superior and postero-superior shoulder joint, the acromion-clavicular joint, capsule and the overlying skin variably [5]. Besides the sensory branches, motor innervation is provided to the supraspinatus and infraspinatus muscles. The nerve itself may be injured as a result of trauma, repetitive overuse, cysts, and tumors and during operative repair around the shoulder [14]. A safe zone, $1 \mathrm{~cm}$ from the glenoid rim has been described to avoid iatrogenic surgical injuries to the SSN [15]. The suprascapular notch has been classified into different categories based on their appearance and their vertical and transverse distances [16]. The presence of a fully calcified TSL or the absence of a notch is considered risk factors for the development of suprascapular neuropathy although there has been no clinical correlation $[16,17]$.

The SSN block has been described using landmark based approaches, CT guidance, ultrasound guidance and electromyography in chronic pain and during regional anesthesia with anatomic landmarks and ultrasound guidance. Many different techniques and sites for the block of the SSN have been described including blocks at the plexus level, in the posterior triangle, at the suprascapular notch, at the spinoglenoid notch and on the superior surface/fossa of the scapular spine $[8,9,18-20]$. In addition to SSN blocks of the articular branch of the circumflex nerve or the axillary nerve have also been described for shoulder pain $[6,21]$.

Diagnostic ultrasound has been utilized for diagnosing various lesions around the SSN [22]. With diagnostic ultrasound, the notch width was found to be $14.14 \pm 4.63 \mathrm{~mm}$ and the skin to notch interval was found to be $41.10 \pm 5.07 \mathrm{~mm}$ on the right among 100 volunteers [23]. Reviewing the ultrasound images showed that our patient had similar dimensions. Ultrasound guidance has been reported for SSN block in a patient with adhesive capsulitis. The authors performed real time injections using $4 \mathrm{ml}$ of levobupivacaine and 80 $\mathrm{mg}$ of triamcinolone acetate with maintained pain relief at 12 weeks [11].

The possibility of SSN entrapment in the clinical differential diagnosis of our patient was not considered because chronologically the pain started following shoulder surgery and any atrophy of the spinatus muscles was not obvious on clinical examination. The fact that he was a weight lifter, a sport predisposing to strenuous overhead activities, may have also contributed to his shoulder pain. He had very limited range of motion of the right shoulder especially external rotation and abduction. He was partly compensating for the decreased range of motion of his shoulder with his scapula and might have developed a pattern of overuse injury. MRI exam did not reveal any specific lesion of the SSN. Being a retrospective review, we were unable to obtain documentation of precise duration of pain relief.

Concerns about pneumothorax and intravascular injection with landmark based techniques exist. Ultrasound guidance may avoid these complications. There were no complications reported with either approaches in our patient.

There have been variations in the amount of injectate used for SSN blocks from $2 \mathrm{ml}$ to $25 \mathrm{ml}[3,4,8,19,24-26]$. The required volume for blocking the SSN was studied in 34 cadavers. The needle was introduced through the "Neviaser portal" which is an area bordered by the clavicle and acromion-clavicular joint laterally, lateral part of the spine posteriorly and the acromion laterally. Comparing the spread of $5 \mathrm{ml}$ and $10 \mathrm{ml}$ of CT contrast agents, the authors performed a CT reconstruction of the injectate spread. They found that the $5 \mathrm{ml}$ injectate spread adequately around the nerve and the $10 \mathrm{ml}$ tended to overflow from the fossa of the spine [24]. In our study with US guidance there was a further significant reduction in the volume of injectate with similar efficacy.

Various injectates and techniques have been used to provide SSN neural blockade. Non specific shoulder pain in 60 patients responded equally to local anesthetic injection of the SSN and subacromial steroid injection [27]. In an earlier prospective blinded randomized study of 26 patients with rheumatoid arthritis, the authors found nearly identical decrease in VAS scores following SSN blocks using bupivacaine and a combination of bupivacaine and methylprednisolone [28]. Similarly, among 9 patients with frozen shoulder a mean decrease in VAS of $8.6 \pm 3.72$ was achieved using only local anesthetics through an anterior landmark based approach with nerve stimulator [8]. It could be postulated that preventing continued afferent signaling from the nociceptive neurons might have provided prolonged pain relief in these patients. Neurolysis of SSN has also been tried with the use of phenol and radiofrequency ablation. Using phenol as a neurolytic (mean change in VAS of 5.9) a longer duration of pain relief for up to 13 weeks was achieved [6]. Following a diagnostic local anesthetic injection to the SSN for shoulder pain, 11 patients underwent pulsed radiofrequency lesioning 
of the SSN. The mean VAS decreased from $7.5 \pm 1.0$ to $2.8 \pm$ 2.6 at their 1 month follow up. It continued to remain low for up to 6 months [29]. Pulsed radiofrequency lesioning was performed in 12 patients with fluoroscopy confirmation of the needle location in yet another study without a comparative or control group. Ten patients showed improvement for up to 6 months [30]. These may be viable options for the non surgical candidate but requires further study.

Intuitively, image guidance for targeting a nerve, would seem to be the best option. But, comparing the landmark based approach to CT guided block of the SSN in 77 patients with chronic shoulder pain of varied etiology, there was no significant difference between the techniques in terms of disability and pain scores [31]. The authors of this single blinded randomized controlled trial commented on the potential for radiation from the exposure to $\mathrm{CT}$ as another disadvantage. But other studies have shown an advantage. In a pilot study of CT guided SSN blocks with steroids, the authors reported a mean decrease in VAS at 30 minutes after the procedure of $3.5 \pm 0.4$ [4]. Our decrease in VAS scores is comparable to these studies. The influence of the cause for the pain cannot be ignored. To our knowledge, no study has yet compared ultrasound to other image guidance techniques.

The limitations of our study are primarily because of its retrospective nature. Only the immediate post procedure VAS was available whereas a prospective study may have covered longer term pain relief. In addition, the shoulder function including range of motion was not assessed. Being a retrospective study, other variables including performance time, patient comfort, and duration of local anesthetic effect were not documented in the notes and hence not available for this study. A prospective study with the patient blinded to the use of ultrasound may provide more valuable information. There is no control for other possible causes for the difference in VAS scores over time. This data is from a "population" of one patient, and is valid for inference about this patient, but these conclusions may not be applicable to other patients. Documentation of duration of pain relief was not precise and suffered from recall bias as with any retrospective study. Since only immediate post procedure VAS was available, the duration of effect of the longer acting local anesthetic in AL was not studied.

In conclusion, this retrospective study suggests that there is possibility for using lesser injectate volume and steroid for blocks of SSN using ultrasound guidance. In addition there is a marginal increase in pain relief with the use of ultrasound guidance. Larger prospective studies are needed to further validate the utility of ultrasound guidance in chronic shoulder pain.

\section{Conflict of Interests}

No conflict of interests for any of the authors.

\section{Fund}

Institutional funding only.

\section{Disclosure}

Submitted in part at the annual fall meeting American Society of Regional Anesthesia and Pain Medicine, 2008.

\section{References}

1. Ostor AJ, Richards CA, Prevost AT, Speed CA, Hazleman BL. Diagnosis and relation to general health of shoulder disorders presenting to primary care. Rheumatology (Oxford) 2005;44(6):800-805.

2. Shanahan EM, Ahern M, Smith M, Wetherall M, Bresnihan B, FitzGerald O. Suprascapular nerve block (using bupivacaine and methylprednisolone acetate) in chronic shoulder pain. Ann Rheum Dis 2003;62(5):400-406.

3. Dahan TH, Fortin L, Pelletier M, Petit M, Vadeboncoeur $\mathrm{R}$, Suissa S. Double blind randomized clinical trial examining the efficacy of bupivacaine suprascapular nerve blocks in frozen shoulder. J Rheumatol 2000;27(6):14641469.

4. Schneider-Kolsky ME, Pike J, Connell DA. CT-guided suprascapular nerve blocks: a pilot study. Skeletal Radiol 2004;33(5):277-282.

5. Ritchie ED, Tong D, Chung F, Norris AM, Miniaci A, Vairavanathan SD. Suprascapular nerve block for postoperative pain relief in arthroscopic shoulder surgery: a new modality? Anesth Analg 1997;84(6):1306-1312.

6. Lewis RN. The use of combined suprascapular and circumflex (articular branches) nerve blocks in the management of chronic arthritis of the shoulder joint. Eur $\mathrm{J}$ Anaesthesiol 1999;16(1):37-41.

7. Shah RV, Racz GB. Pulsed mode radiofrequency lesioning of the suprascapular nerve for the treatment of chronic shoulder pain. Pain Physician 2003;6(4):503506.

8. Wassef MR. Suprascapular nerve block. A new approach for the management of frozen shoulder. Anaesthesia 1992;47(2):120-124.

9. Risdall JE, Sharwood-Smith GH. Suprascapular nerve block. New indications and a safer technique. Anaesthesia 1992;47(7):626.

10. Karatas GK, Meray J. Suprascapular nerve block for pain relief in adhesive capsulitis: comparison of 2 different techniques. Arch Phys Med Rehabil 2002;83(5):593597.

11. Harmon D, Hearty C. Ultrasound-guided suprascapular nerve block technique. Pain Physician 2007;10(6):743746. 
12. Moore DC. Block of the suprascapular nerve. In Thomas CC, ed. Regional nerve block, 4th ed. Springfield, 1979;9:300-303.

13. Aszmann OC, Dellon AL, Birely BT, McFarland EG. Innervation of the human shoulder joint and its implications for surgery. Clin Orthop Relat Res 1996;(330):202207.

14. Cummins CA, Messer TM, Nuber GW. Suprascapular nerve entrapment. J Bone Joint Surg Am 2000;82(3):415424.

15. Bigliani LU, Dalsey RM, McCann PD, April EW. An anatomical study of the suprascapular nerve. Arthroscopy 1990;6(4):301-305.

16. Natsis K, Totlis T, Tsikaras P, Appell HJ, Skandalakis P, Koebke J. Proposal for classification of the suprascapular notch: a study on 423 dried scapulas. Clin Anat 2007;20(2):135-139.

17. Dunkelgrun M, Iesaka K, Park SS, Kummer FJ, Zuckerman JD. Interobserver reliability and intraobserver reproducibility in suprascapular notch typing. Bull Hosp Jt Dis 2003;61(3-4):118-122.

18. Dangoisse MJ, Wilson DJ, Glynn CJ. MRI and clinical study of an easy and safe technique of suprascapular nerve blockade. Acta Anaesthesiol Belg 1994;45(2):4954.

19. Matsumoto D, Suenaga N, Oizumi N, Hisada Y, Minami A. A new nerve block procedure for the suprascapular nerve based on a cadaveric study. J Shoulder Elbow Surg 2009;18(4):607-611.

20. Roark GL. Suprascapular nerve block at the spinoglenoid notch. Reg Anesth Pain Med 2003;28(4):361-362.

21. Checcucci G, Allegra A, Bigazzi P, Gianesello L, Ceruso M, Gritti G. A new technique for regional anesthesia for arthroscopic shoulder surgery based on a suprascapular nerve block and an axillary nerve block: an evaluation of the first results. Arthroscopy 2008;24(6):689-696.

22. Martinoli C, Bianchi S, Pugliese F, Bacigalupo L, Gaug- lio C, Valle M, Derchi LE. Sonography of entrapment neuropathies in the upper limb (wrist excluded). J Clin Ultrasound 2004;32(9):438-450.

23. Yucesoy C, Akkaya T, Ozel O, Comert A, Tuccar E, Bedirli N, Unlu E, et al. Ultrasonographic evaluation and morphometric measurements of the suprascapular notch. Surg Radiol Anat 2009;31(6):409-414.

24. Feigl GC, Anderhuber F, Dorn C, Pipam W, Rosmarin W, Likar R. Modified lateral block of the suprascapular nerve: a safe approach and how much to inject? A morphological study. Reg Anesth Pain Med 2007;32(6):488494.

25. Barber FA. Suprascapular nerve block for shoulder arthroscopy. Arthroscopy 2005;21(8):1015.

26. Emery P, Bowman S, Wedderburn L, Grahame R. Suprascapular nerve block for chronic shoulder pain in rheumatoid arthritis. BMJ 1989;299(6707):1079-1080.

27. Taskaynatan MA, Yilmaz B, Ozgul A, Yazicioglu K, Kalyon TA. Suprascapular nerve block versus steroid injection for non-specific shoulder pain. Tohoku J Exp Med 2005;205(1):19-25.

28. Gado K, Emery P. Modified suprascapular nerve block with bupivacaine alone effectively controls chronic shoulder pain in patients with rheumatoid arthritis. Ann Rheum Dis 1993;52(3):215-218.

29. Liliang PC, Lu K, Liang CL, Tsai YD, Hsieh CH, Chen HJ. Pulsed radiofrequency lesioning of the suprascapular nerve for chronic shoulder pain: a preliminary report. Pain Med 2009;10(1):70-75.

30. Kane TP, Rogers P, Hazelgrove J, Wimsey S, Harper GD. Pulsed radiofrequency applied to the suprascapular nerve in painful cuff tear arthropathy. J Shoulder Elbow Surg 2008;17(3):436-440.

31. Shanahan EM, Smith MD, Wetherall M, Lott CW, Slavotinek J, FitzGerald O, Ahern MJ. Suprascapular nerve block in chronic shoulder pain: are the radiologists better? Ann Rheum Dis 2004;63(9):1035-1040. 